\title{
3D Position and Velocity Vector Computations of Objects Jettisoned from the International Space Station Using Close-Range Photogrammetry Approach
}

\author{
Valeri Papanyan ${ }^{\mathrm{a}^{*}}$, Edward Oshel ${ }^{\mathrm{a}}$, and Daniel Adamo ${ }^{\mathrm{b}}$ \\ ${ }^{a}$ Image Science and Analysis Group, NASA-JSC/KX; Jacobs Technology/ESCG, P.O. Box 58447, \\ Mail Code: JE36-2, Houston, TX 77258-8447 \\ ${ }^{\mathrm{b}}$ United Space Alliance, LLC, Johnson Space Center-DM3/Orbit Dynamics Branch,
}

\begin{abstract}
Measurement of the jettisoned object departure trajectory and velocity vector in the International Space Station (ISS) reference frame is vitally important for prompt evaluation of the object's imminent orbit. We report on the first successful application of photogrammetric analysis of the ISS imagery for the prompt computation of the jettisoned object's position and velocity vectors. As post-EVA analyses examples, we present the Floating Potential Probe (FPP) and the Russian "Orlan” Space Suit jettisons, as well as the near-real-time (provided in several hours after the separation) computations of the Video Stanchion Support Assembly Flight Support Assembly (VSSA-FSA) and Early Ammonia Servicer (EAS) jettisons during the US astronauts space-walk. Standard close-range photogrammetry analysis was used during this EVA to analyze two on-board camera image sequences down-linked from the ISS. In this approach the ISS camera orientations were computed from known coordinates of several reference points on the ISS hardware. Then the position of the jettisoned object for each time-frame was computed from its image in each frame of the video-clips. In another, "quick-look" approach used in near-real time, orientation of the cameras was computed from their position (from the ISS CAD model) and operational data (pan and tilt) then location of the jettisoned object was calculated only for several frames of the two synchronized movies.
\end{abstract}

Keywords: Photogrammetry, International Space Station, jettisons, image analysis.

\section{INTRODUCTION}

Throughout continuing operations of the International Space Station (ISS) its crew has to jettison an increasing number of obsolete items, occupying costly on-orbit space, and even potentially hazardous equipment. However, this procedure poses considerable hazard due to high relative kinetic energy of the objects and a possibility of their recontact on subsequent orbits. This possibility is high because any jettison relies on a crew-member imparted impulse, which is highly dispersed in spite of rigorous planning and extensive training. It is the case for both small and large object jettisons. An example of a small object jettison is the so called "Golf-Ball Experiment" when the ball was driven from the Russian segment of the ISS. The ball's actual trajectory was in almost the opposite direction from which it was planned. In the opposite case, the heaviest jettison object was the Early Ammonia Server (EAS) jettison that was successfully accomplished by astronaut Clay Anderson (see section 4-c). He has a background in trajectory analysis (in fact, he gave a presentation to the 1978 astronaut class on Shuttle rendezvous [1]) and in spite of his long hours of extensive training in the JSC Training Facility (zero-gravity training environment), the EAS (as well as VSSA) was directed much more towards Zenith (away from the Earth, it was down for the astronaut) than was planned. It is obvious that any jettisoned object imposes a life-threatening danger for the Station in the case of its recontact. Therefore, as the human element is present at the last and most decisive phase of any jettison during EVA (Extra Vehicular Activity or crew-walk in open space), fast and reliable determination of the jettisoned object trajectory is extremely important.

\footnotetext{
* Phone: 281-483-5144, Fax: 281-483-7303; e-mail: valeri.papanyan-1@nasa.gov
} 
Experience with previous ISS jettisons indicates that it may be days after the jettison before reliable orbit determination data becomes available to the Mission Control Center (MCC) from the ground (e.g., USSTRATCOM radars and optical sensors) and it may be even impossible if the object is small (e.g., for the Golf Ball jettison). Consequently, for the prompt evaluation the object's trajectory, knowledge of its departure 3D velocity vector and trajectory relative to the ISS is vitally important.

We report in this paper on the first successful application of photogrammetric analysis for the timely computation of the jettisoned object's trajectory and velocity from on-orbit imagery. We will describe the post-EVA analyses examples, such as Floating Potential Probe (FPP) and Space Suit jettisons (sections 4a and 4b), as well as the real-time (several hours after the separation event) computation of the VSSA and EAS jettisons (sections 4-c and 4-d). Standard closerange photogrammetry analysis was used based on two on-board camera image sequences down-linked from the ISS. In this approach the ISS truss camera positions were defined from the ISS CAD model. The camera orientation was computed from the image coordinates of several reference points on the ISS hardware. Then position of the jettisoned object was computed from its image in each frame of the video-clips. We also describe a "quick-look" approach, used in real time, when orientation of the cameras was computed from their operational data on their pan and tilt and then location of the jettisoned object was calculated by intersection only for two images of the two synchronized movies (section 3).

\section{PHOTOGRAMMETRY ON THE ISS}

"Photogrammetry is the science and art of determining the size and shape of objects as a consequence of analyzing images recorded on film or electronic media." [2] The word 'science' is obvious as it implies applications of mathematics, physics, and engineering. However, 'art' must not be overlooked as good results can only be produced from suitable imagery, so photography and movie-making skills are also required. For the trajectory computation, which is concern of this paper, we need to calculate 3D position of an object's center of mass in each frame of two or more movies, where the motion was recorded, therefore, it is called often 'Videogrammetry' or '4.4 Photogrammetry'.

In principle, we can track only one point on the object in the case of pure translational motion. However, it is not always possible due to change of illumination and fixed camera positions. If a large object is jettisoned, it may rotate, so that for computation its 6D position (including roll, pitch, and yaw) several points on the object (target points) should be tracked throughout the video-clip.

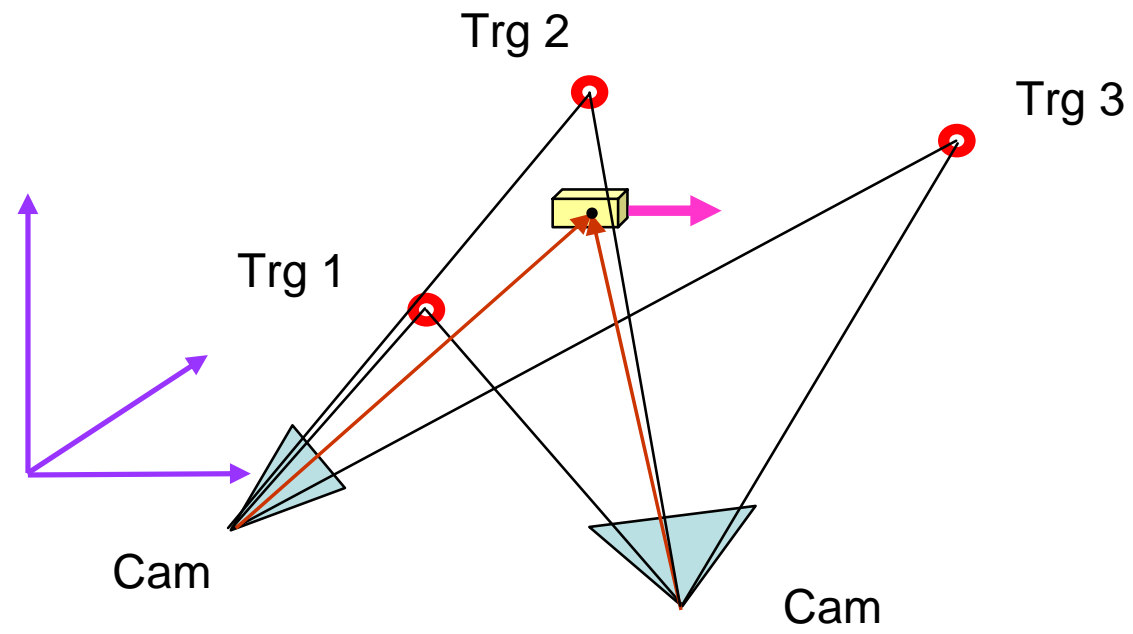

Fig. 1. Perspective geometry (convergent configuration) used for photogrammetry calculations.

In photogrammetry computation of an object's coordinates is a two-step process: first, the camera's position and orientation, so called, external parameters, in the given system of coordinates must be determined ('resection' of the 
cameras), and secondly the object's 3D coordinates must be calculated from each of the two (at least) images, that is intersection computation or triangulation. It is obvious that for calculating the object's 3D coordinates from its two dimensional image position we need at least two cameras. As the camera position and orientation contains six degrees of freedom, at least three reference targets with well defined coordinates are needed in the FOV (Field Of View) of each camera. This can be employed in practice if the lens focal length and distortion parameters (so called 'internal parameters') are known. This, first step, is called 'resection' and, obviously, more targets (dispersed all around the FOV) are essential for better accuracy of the calculations. Usually, this is not the case for on-orbit photogrammetry, where it is difficult, and sometimes impossible, to locate ISS hardware in the FOV during the eventual tracking, not to mention inadvertent jettison situations, when trajectory data are requested after the event happened. Also, remote control of the ISS cameras that are stationed in open space does not give an opportunity to know their zoom and attitude angles with needed accuracy, so that plenty of reference targets would improve knowledge of the internal parameters. The two steps (resection and intersection) nowadays are combined in simultaneous computations with the best adjustment using the least squares method [3]. In the case of videogrammetry these calculations must be performed for each frame of the video clip that yields necessity of intense computations to be performed, which is a difficult task to perform in real time even today. Essentially, the two (or more) cameras must be synchronized, at least timing of each image field must be recorded, so that the software can interpolate motion taking into account the time shift between the cameras' exposures.

\section{INTERSECTION METHOD APPLICATION}

During the first part of its trajectory, when the jettisoned object is still in the field of view from the ISS cameras, we can neglect the orbital displacement of the object and the ISS, and assume that the jettisoned object's motion has constant velocity. It makes possible a fast assessment of the object's velocity components relative to the ISS because it should be enough to measure its position in two points of the trajectory. This method was realized by writing a short script in MATLAB, and successfully used in real time during the EAS and VSSA jettisons (see Quick Look column in Tables 2 and 3 below).

The method uses apriori "as designed" models of the ISS and the camera mount structures to provide initial estimates of the camera positions (projection center coordinates). The pan and tilt of the cameras are noted in "real time" and used to compute a more accurate position and orientation of the cameras in the ISS coordinate system. The measured image coordinates are combined with the projection center to produce a Line-of-Sight (LOS) vector from the projection center towards the object. A 3D intersection of the two LOS vectors is computed to produce an estimate of the location of the object. The position is computed at two different times and differenced to yield an estimated velocity of the object.

\section{ISS JETTISON EXAMPLES}

\subsection{Floating Potential Probe (FPP) jettison}

The Floating Potential Probe (FPP) was designed to measure the Station's electrical potential and compare it to the surrounding plasma. It was mounted atop the Z-truss between the station's solar wings (UFL1.1 configuration). During one of the spacewalks (Extra-Vehicular Activity), of the STS-114 mission its crew recorded imagery of the FPP. After ground analysis of the imagery, ISS team identified an anomaly with a structural fastener and a handle release button on the device. Analysis of images revealed that two FPP fasteners backed out from their normally engaged position. That raised concerns that the fasteners could become detached and, perhaps, cause damage. Subsequently, the Station program management decided to jettison the FPP. The actual jettison was performed during the US EVA 4 by the ISS Expedition 12 Commander William MacArthur on November 7, 2005. [4] MacArthur was attached to the Articulating Portable Foot Restraint (APFR) and was positioned on the Z-Truss facing aft between the solar arrays. To ensure the FPP safe departure, it had to be jettisoned retrograde at a minimum speed of $0.5 \mathrm{f} / \mathrm{s}$ with a smooth motion, in the direction of 30 degrees zenith and 10 degrees port (relative to the ISS velocity vector), approximately. Following jettison, the FPP should have cleared the Station, de-orbited, and burned in the Earth's atmosphere. After the jettison took place, a request was made by the MER (Mission Evaluation Room) for the Image Science and Analysis Group (ISAG) to measure velocity vector of the departing FPP from the imagery taken during the spacewalk. 


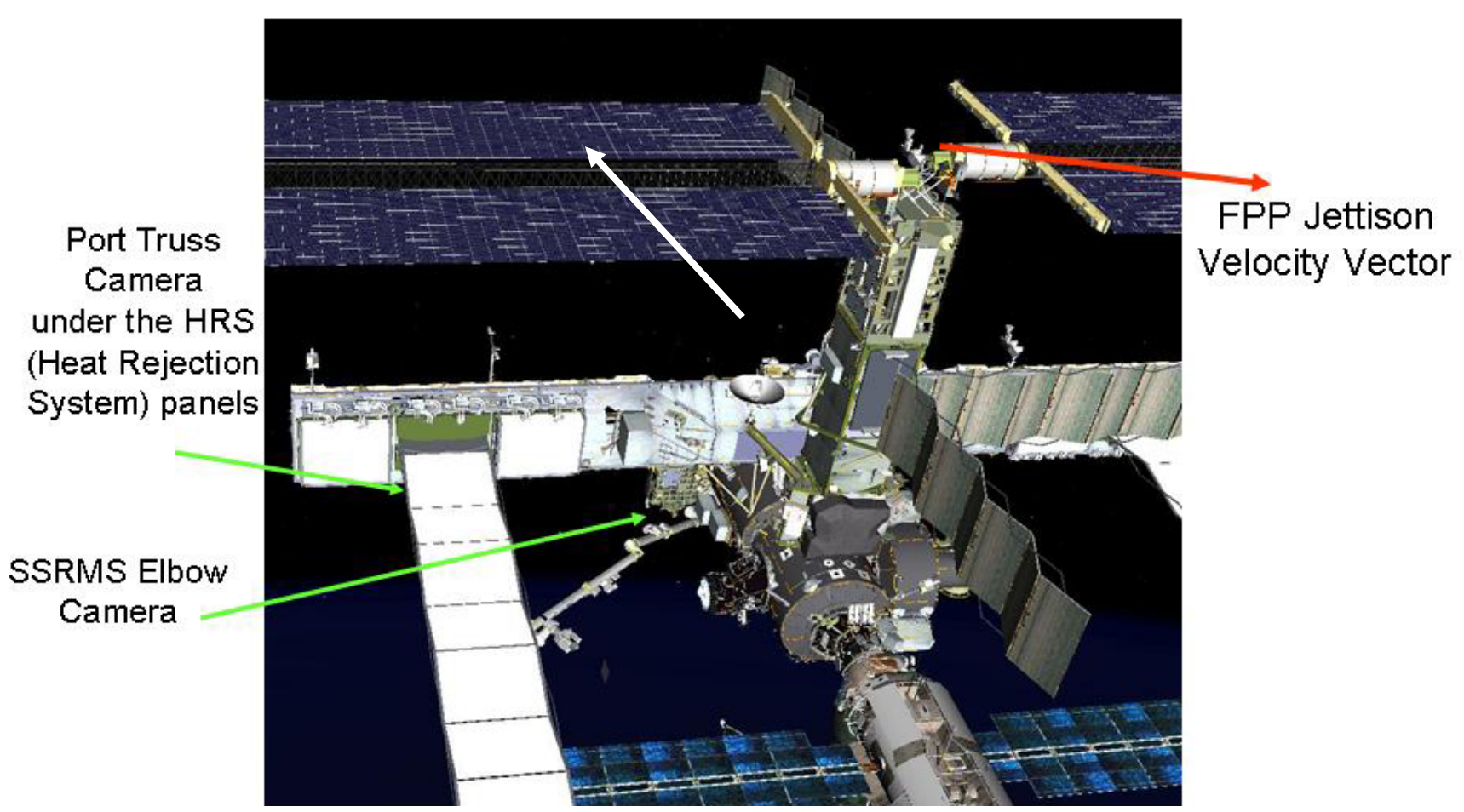

Fig. 2. FPP was jettisoned from the "top" of the Z-truss in the ISS-relative direction shown by the red arrow. Two cameras recorded the jettison (not taking into account the astronauts helmet cameras); the SSRMS (Space Station Remote Manipulator System) elbow camera and the truss port camera, which is not visible here underneath the port HRS (Heat Rejection System) panels. White arrow shows the geocentric ISS vector of velocity.

Two video-clips from the SSRMS (Space Station Remote Manipulator System) elbow camera and the truss port camera were analyzed. The astronauts' helmet cameras, which also recorded the jettison, were not suitable for photogrammetric analysis because of their unstable position.

This analysis was complicated by the fact that the cameras had no mutual synchronization and no frame encryption was available (because photogrammetry application was not planned beforehand). Therefore, visual synchronization was performed by comparing of the FPP attitude-position in reference to its trajectory. To estimate error of this method, trajectory was also computed not only for this time-sync but also with shifted by several frames $( \pm 3, \pm 6, \pm 9$, and \pm 12 frames) video sequences. Shift in synchronization by $\pm 0.1 \mathrm{sec}$ (which was \pm 3 frames), yielded the trajectory coordinates shift by the distance of $( \pm) 0.25 \mathrm{~m}=0.8$ feet, which FPP covers during this time interval.

It was possible to use 28 reference points in the ISS Analysis Coordinate System (ISSACS) $\left[{ }^{\dagger}\right]$ for resection (calculation of the camera position, attitude, and internal parameters) of the Port Truss camera and only 9 points for resection of the SSRMS Elbow camera. Calculated by the Trackeye software [5] average (over 210 frames) position of the Port Camera appeared to be very close to its truth location, the difference was only several inches for each coordinate. However, the result for the Starboard camera was not so encouraging. Nevertheless, computations of the FPP centroid (geometrical center of the visible image/spot) 3D coordinates in each frame were successful.

The FPP departure speed over 210 frames was found to be $2.4( \pm 0.3) \mathrm{m} / \mathrm{s}=8( \pm 1)$ feet/sec. Hence, the resulting speed is much above the $0.5 \mathrm{f} / \mathrm{s}$ required minimum speed. Also of importance is that computed components of the velocity vector showed that it is well inside the safety cone (see Fig. 4).

\footnotetext{
${ }^{\dagger}$ ISSACS (also called Space Station Reference Frame - SSREF) has its origin in the center of the Integrated Truss Structure with Xaxis parallel to the module cluster and directed forward (US Lab module), positive Y-axis in the starboard direction, and the positive Z-axis in direction of Nadir.
} 

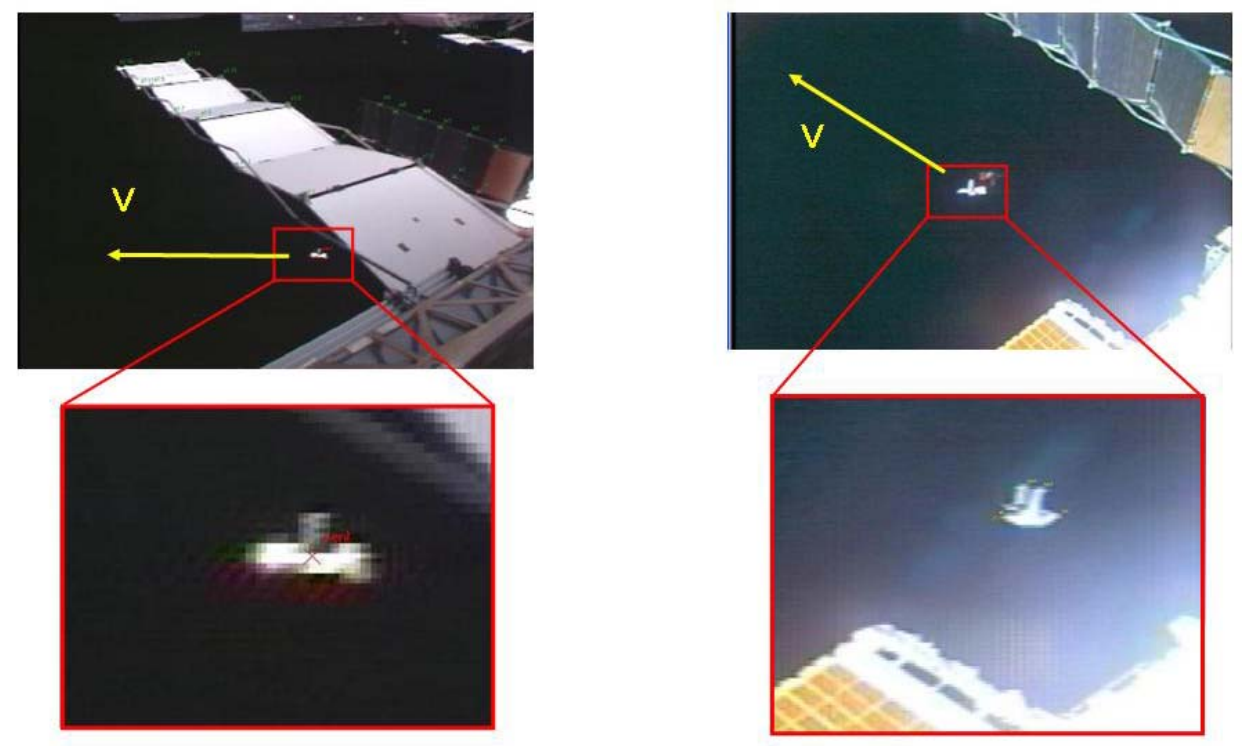

Fig. 3. Synchronization of the frames from the Port Truss camera (left) and the SSRMS camera (right).
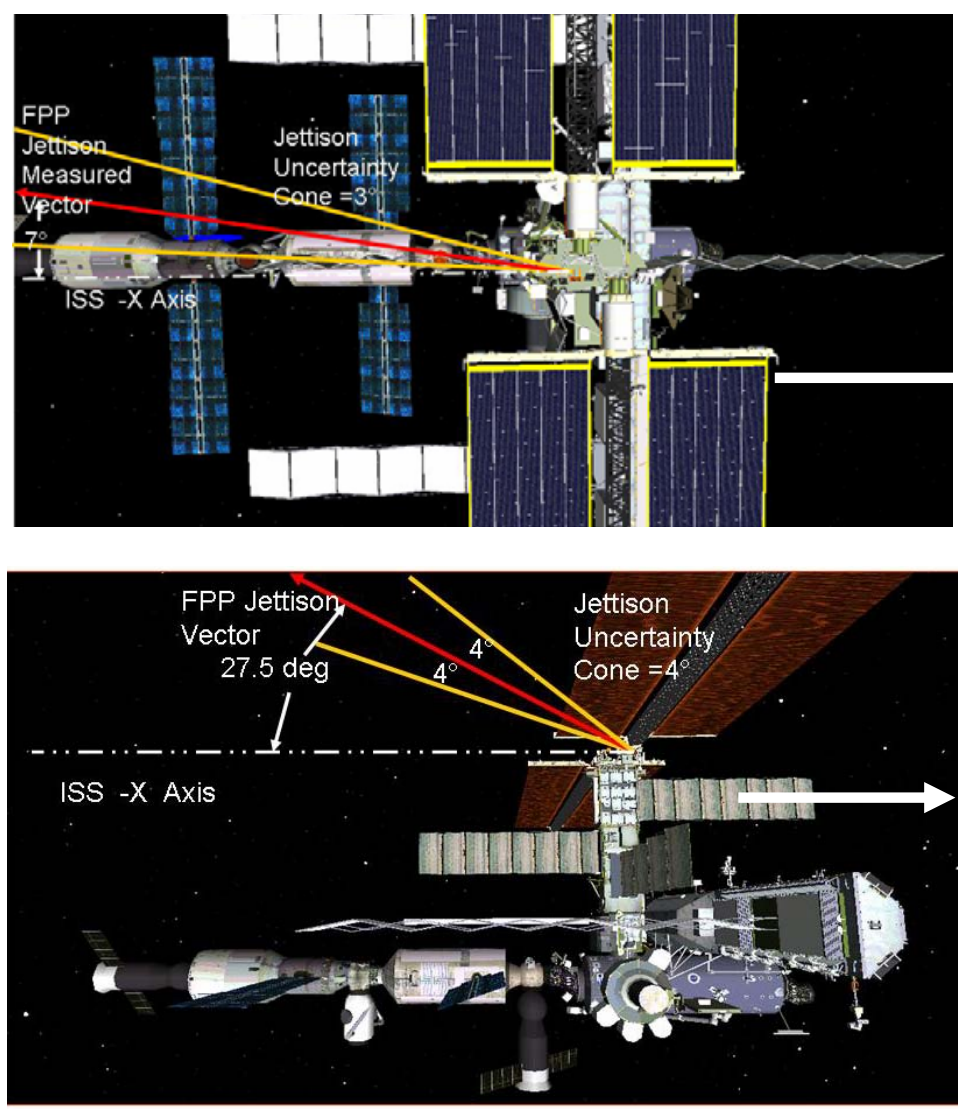

Fig. 4. The calculated FPP jettison velocity direction with the measurements uncertainty cone shown for the view looking nadir onto the ISS (top) and the port side of the ISS (bottom). 


\section{2. “Orlan” Space Suit jettison}

So called "Space Suit Satellite" (nicknamed Ivan Ivanovich) was jettisoned from the ISS by the Expedition 12 crew during EVA on February 3, 2006. It was released by Valery Tokarev and William McArthur towards aft and nadir from the starboard side of the Docking Compartment, located between the Russian Service Module 'Zvezda' and the Functional Cargo Block (FGB) [6]. The Space Suit was a decommissioned Russian "Orlan” spacesuit equipped with a ham radio transmitter and a compact disk containing messages and images from students around the world. The suit transmitted a ham radio signal for several days before its batteries lost power. Later it was burned up upon reentering the Earth's atmosphere.

Subsequent to the jettison an analysis of the actual release trajectory was requested, as well as the closest distance estimation from the spacesuit to the ISS hardware. The videos provided for the analysis were recorded by the ISS Port and Starboard Truss cameras.

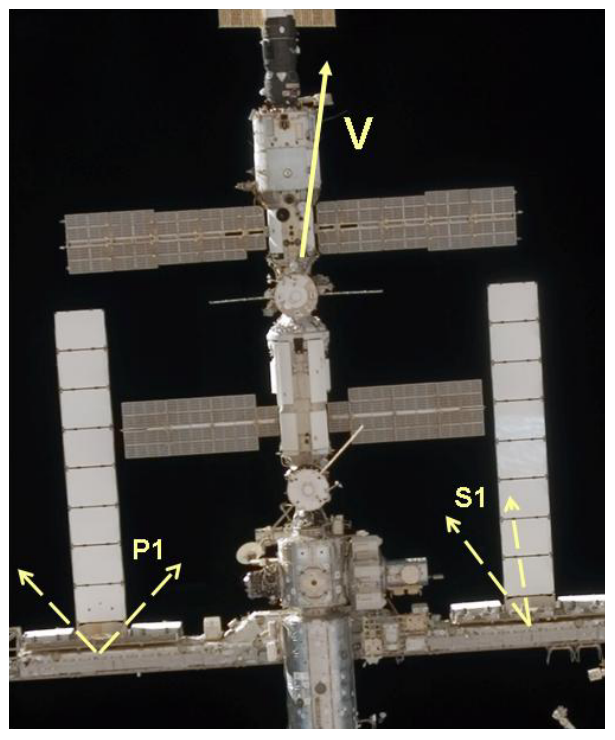

Fig. 5. Nadir view of the Space Station with velocity vector of the jettisoned Space Suit and approximate FOV of the Port and Starboard cameras.

Selected frames from the Port and Starboard camera video-clips are shown on Fig. 6 and 7. Starboard camera was zoomed too much onto the worksite, at the same time the Port camera had too wide FOV (Field Of View), so that the Space Suit's image occupied only several pixels on the image. Therefore, the views were not favorable for a high-quality photogrammetry analysis. However, the Suit trajectory and departure velocity were obtained over the time span of 21 seconds. For this analysis 25 reference points in the Port camera view were sufficient for calculating its position and orientation with satisfactory accuracy (disagreement with the truth data was only several inches at about 1300 inches distance to the targets). In case of the Starboard camera, however, from 12 reference points that were possible to use throughout the whole tracking interval, 11 were in one plane (on the FGB/Functional Cargo Block solar array) and only one point (the Magnetometer package) was out of this plane. The other three points, on the 'Pirs' Docking Module hardware, have been in the FOV for a short period of time $(3.5 \mathrm{sec})$ only in the beginning of the video-clip. Consequently, position of the Starboard camera was not defined well enough yielding rather large uncertainty for the absolute coordinates results.

The Space Suit trajectory 3D coordinates' calculation results in the ISSACS (ISS Analysis Coordinate System) are illustrated on Fig. 8. The Magnetometer Y-coordinate plot is also reproduced (in black) for visualization of the error magnitude. The bottom chart shows the Suit's center, helmet and boots distance from the Magnetometer, where the vertical bar shows the closest center position - it was only 70 inches. It is obvious that the Space Suit passed too close to 
the Magnetometer, presenting a safety hazard, which was not realized during the planning stage of this jettison. The Space Suit departure velocity components are given in Table 1, its speed was found to be approximately 12 in/s.

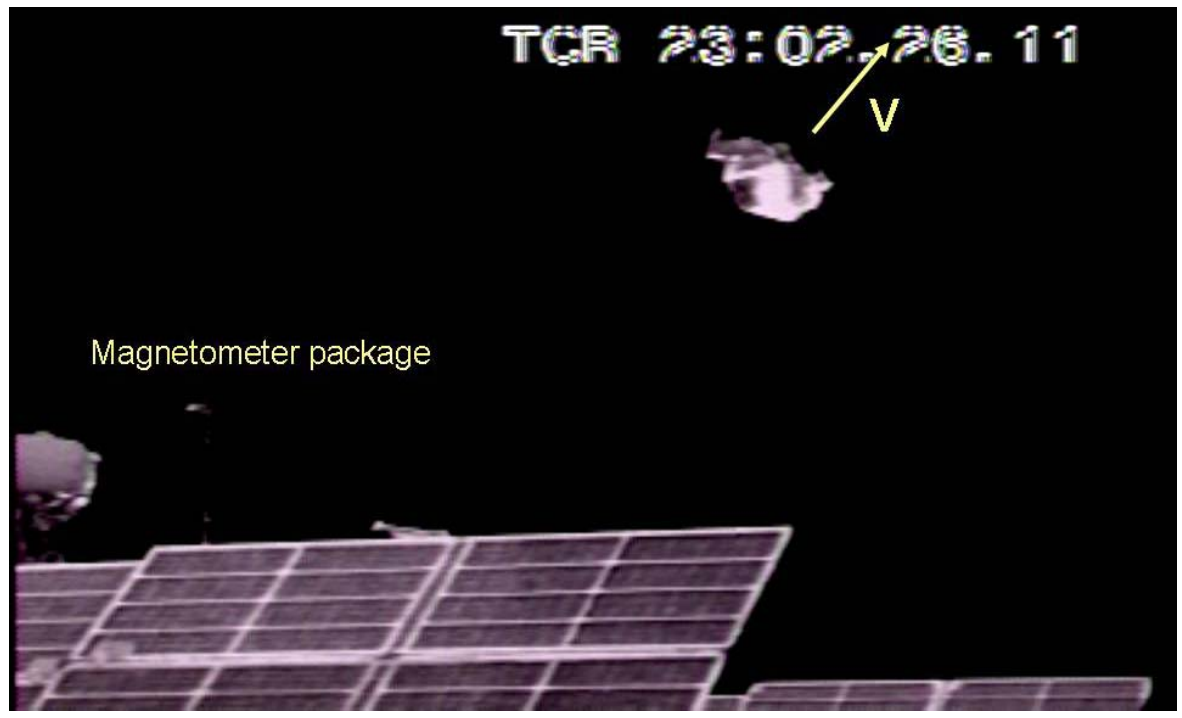

Fig. 6. Starboard camera view at 26 seconds after the Space Suit separation.

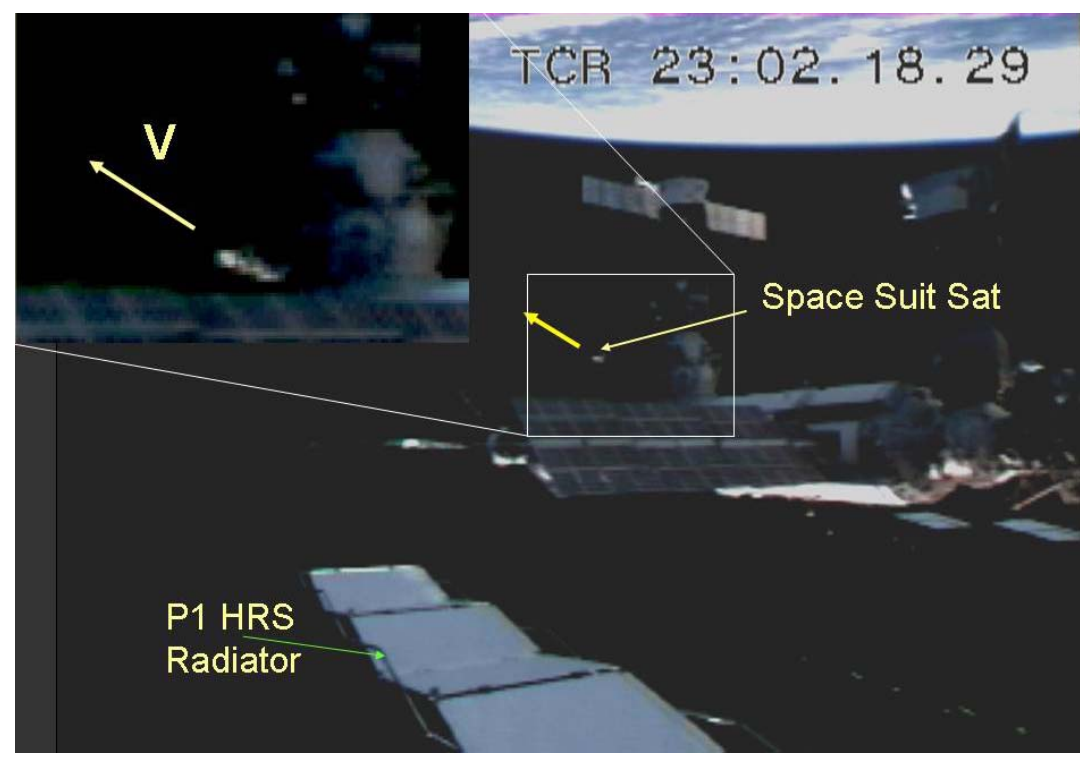

Fig. 7. Port camera view at 18 seconds after the Space Suit separation. In zoom window pixilation of the Space Suit image is obvious. Arrows indicate Suit velocity with respect to ISS.

Table 1 . Space Suit velocity computation results.

\begin{tabular}{|l|c|c|c|c|c|c|c|}
\hline & $\begin{array}{c}\text { Vx, } \\
\text { in/s }\end{array}$ & $\begin{array}{c}\text { Vy, } \\
\text { in/s }\end{array}$ & $\begin{array}{c}\text { Vz, } \\
\text { in/s }\end{array}$ & $\begin{array}{c}\text { Speed, } \\
\text { in/s }\end{array}$ & $\begin{array}{c}\text { Angle-X, } \\
\text { deg }\end{array}$ & $\begin{array}{c}\text { Angle-Y, } \\
\text { deg }\end{array}$ & $\begin{array}{c}\text { Angle-Z, } \\
\text { deg }\end{array}$ \\
\hline $\begin{array}{l}\text { 9-point calculation then } \\
\text { averaged over all frames }\end{array}$ & -11.03 & -0.71 & 4.71 & 12.02 & 23.3 & 93.4 & 66.9 \\
\hline $\begin{array}{l}\text { Slope trends from raw } \\
\text { coordinates tracked }\end{array}$ & -11.10 & -0.56 & 4.38 & 11.95 & 21.7 & 92.7 & 68.5 \\
\hline
\end{tabular}




\begin{tabular}{|l|l|l|l|l|l|l|l|}
\hline Standard Deviation & 0.04 & 0.02 & 0.03 & & & & \\
\hline
\end{tabular}
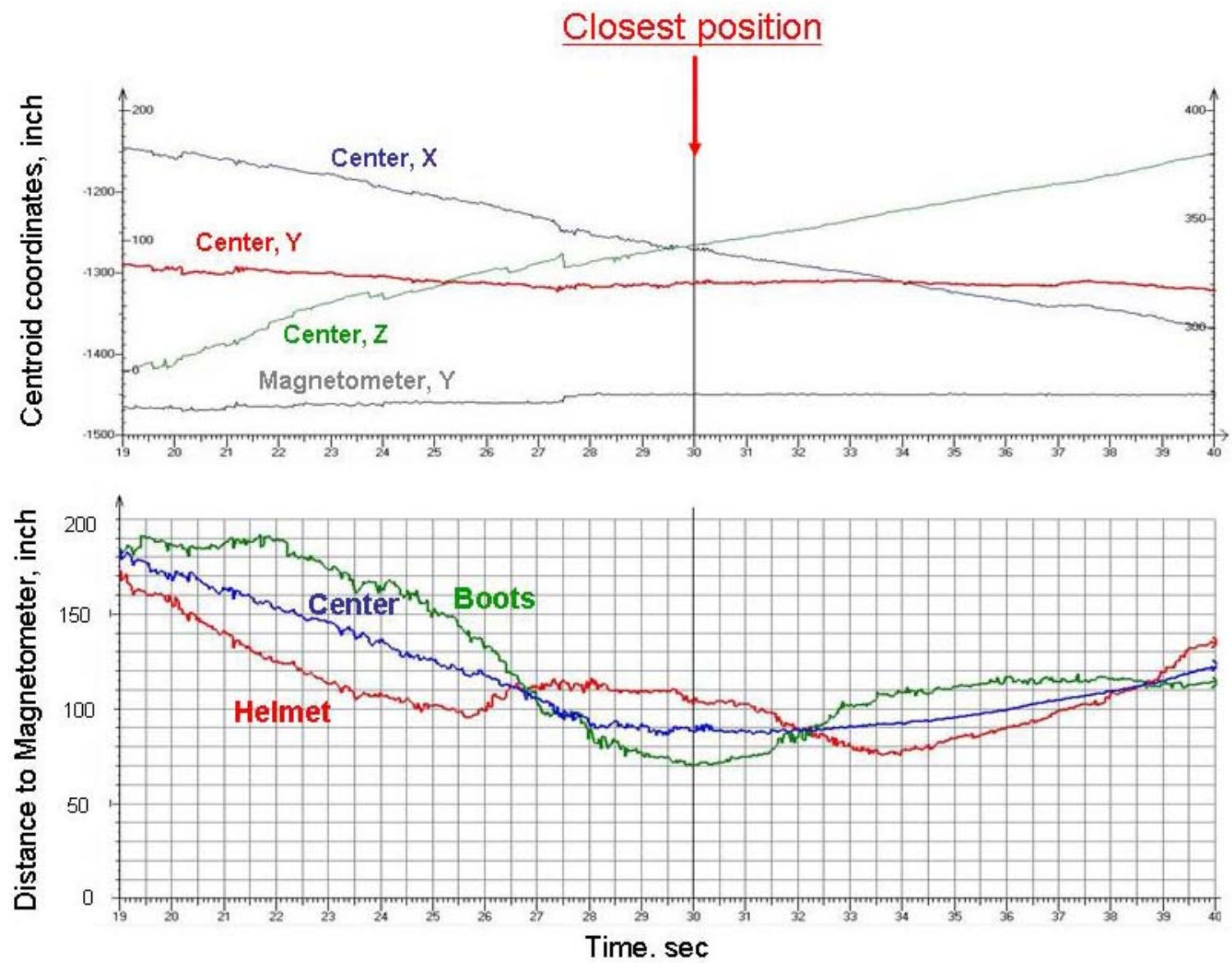

Fig. 8. Calculated plots of the Space Suit centroid 3D coordinates in the ISS system is shown on the top; distances of its center, boots, and helmet from the Magnetometer package are shown on the bottom chart.

\subsection{Early Ammonia Server (EAS) jettison}

The Early Ammonia Servicer (EAS) was installed on the P6 truss during STS-105 mission in August 2001, as an ammonia replenishment reservoir for ISS cooling, if a leak had occurred. The EAS was designed for a maximum 5 year on-orbit lifetime; its mass was $650 \mathrm{~kg}$, and dimensions 2.43 × 1.16 × $2.73 \mathrm{~m}$ (shown in Fig. 9). It was never used, and was no longer needed after the permanent cooling system was activated in December 2006. The EAS had to be removed, to open space for the P6 truss that should be installed to the end of the station's main truss by the next Shuttle crew (STS118 in August 2007). The operation of removal and jettison was performed during the ISS crew EVA on July 23, 2007 by US astronaut Clay Anderson and Russian cosmonaut Fyodor Yurchikhin. They detached the EAS from the truss and Clay Anderson, who was fastened to Articulating Portable Foot Restraint (APFR) attached to the SSRMS (Space Station Remote Manipulation System) grappled the EAS and held it during the SSRMS maneuver to the jettison position, which was as far from the ISS hardware as possible (shown in Fig. 10). Then, after a pause, he pushed the unit away [7]. Before this complicated operation began, the ISS was oriented as flying "backward” so that now the astronaut's thrust will force the EAS to acquire $\Delta \mathrm{V}$ in retrograde direction (opposite to the ISS velocity vector) that will put it on a lower orbit with respect to the Station. A subsequent reboost by Progress thrusters will change the station's orbit to a higher one that will 
provide safety clearance from the slowing down EAS. Therefore, it was extremely important to measure the EAS velocity relative to the ISS as quickly as possible, to make a decision if a second reboost will be needed to ensure the ISS safety.

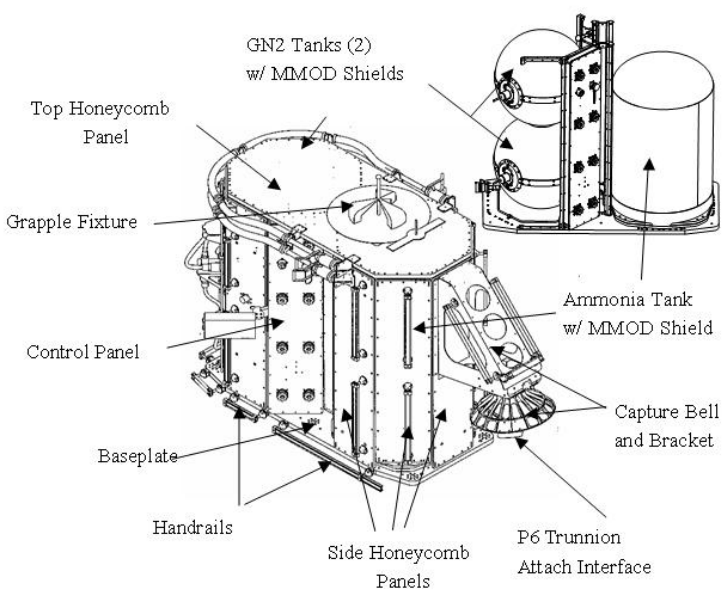

Fig. 9. EAS description.

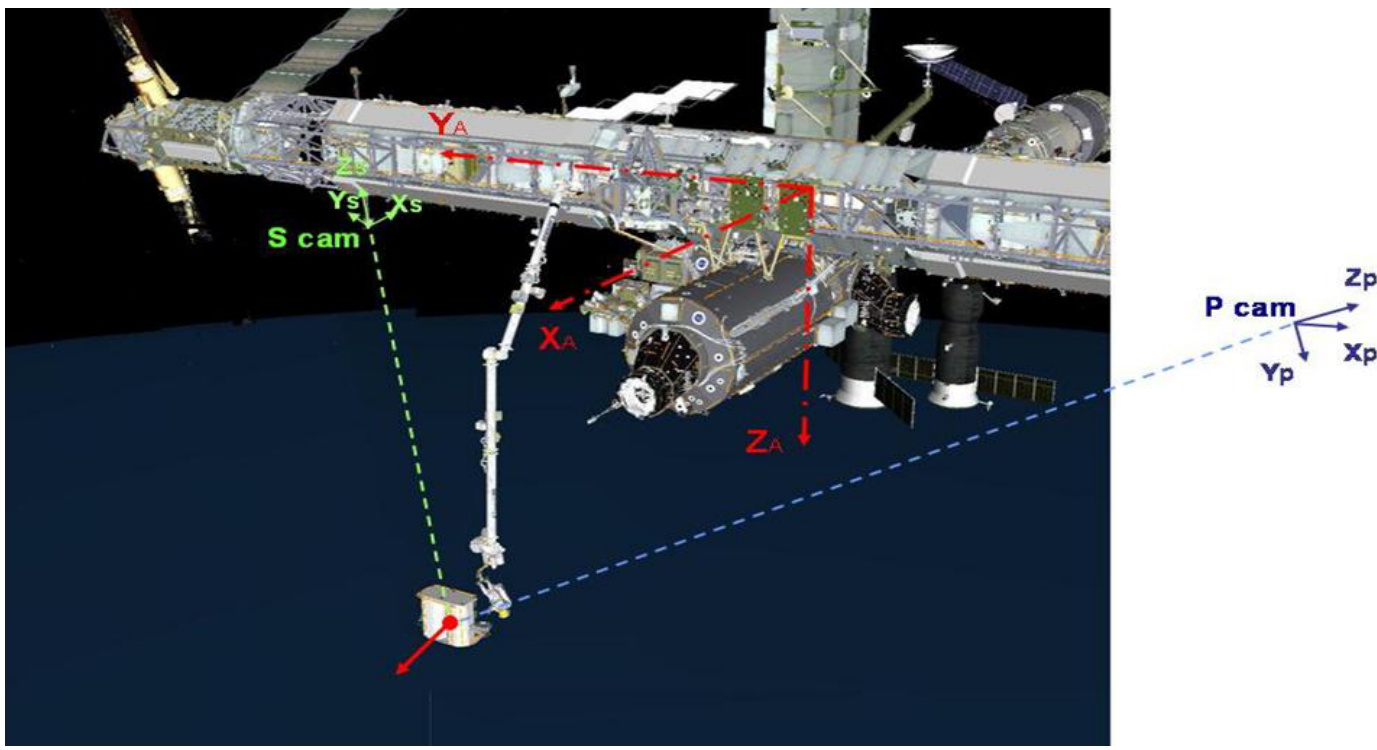

Fig. 10. Planned and performed jettison position shown on the model graphics with the truss camera systems of coordinates in green and blue and the ISSACS axes in red dashed. Red arrow emanating from the EAS center indicates the planned vector of jettison velocity.

The EAS jettison was planned long before it happened. The crewmember was supposed to jettison the EAS (as well as VSSA, see the next section) parallel to the ISS vector of velocity but in the opposite direction within $\pm 30^{\circ}$ (safety structural clearance cone) with speed at least $5 \mathrm{~cm} / \mathrm{s}$. These requirements were based on the ballistic analysis that in the expected atmospheric density conditions which showed that at $5 \mathrm{~cm} / \mathrm{s} \quad \mathrm{V}$ the worst case scenario had the EAS returning to the vicinity of the ISS in 19 hours. Required $\Delta \mathrm{V}$ was therefore raised up to $15 \mathrm{~cm} / \mathrm{s}$, to ensure a safe departure because in this case 63 hours (worst case return) will guarantee time for reboost of the ISS to avoid recontact. This expectation was based on precision air bearing floor (PABF) testing, EVA analysis, and past operational experience. Clay Anderson was extensively trained in the zero-g environment (JSC Sonny Carter Training Facility [8]) for these manipulations with 
a large, refrigerator size, unit. Any error could cause a significant damage to the Station hardware if EAS would contact with the station during the jettison or on the first several orbits.

It was supposed that the Space Surveillance Network (SSN) would be able to acquire and track the EAS tens of hours or, maybe, days after the event. An avoidance maneuver could not be planned and carried out so late. For prompt evaluation of the first EAS orbits, measurement of the jettisoned object velocity vector was recognized as extremely important. This task was completed by tracking the first minutes after separation with the two ISS Truss cameras, and calculating the velocity vector in near real time in the Image Science and Analysis Laboratory at JSC. During the EVA visual observation it was already obvious that EAS (as well as the VSSA, see the next section) was jettisoned not exactly in the planned direction, but much shifted to the Zenith (see Fig. 11). Therefore, accurate computation of the initial velocity in ISSACS (ISS Analysis Coordinate System) turned out to be even more important.
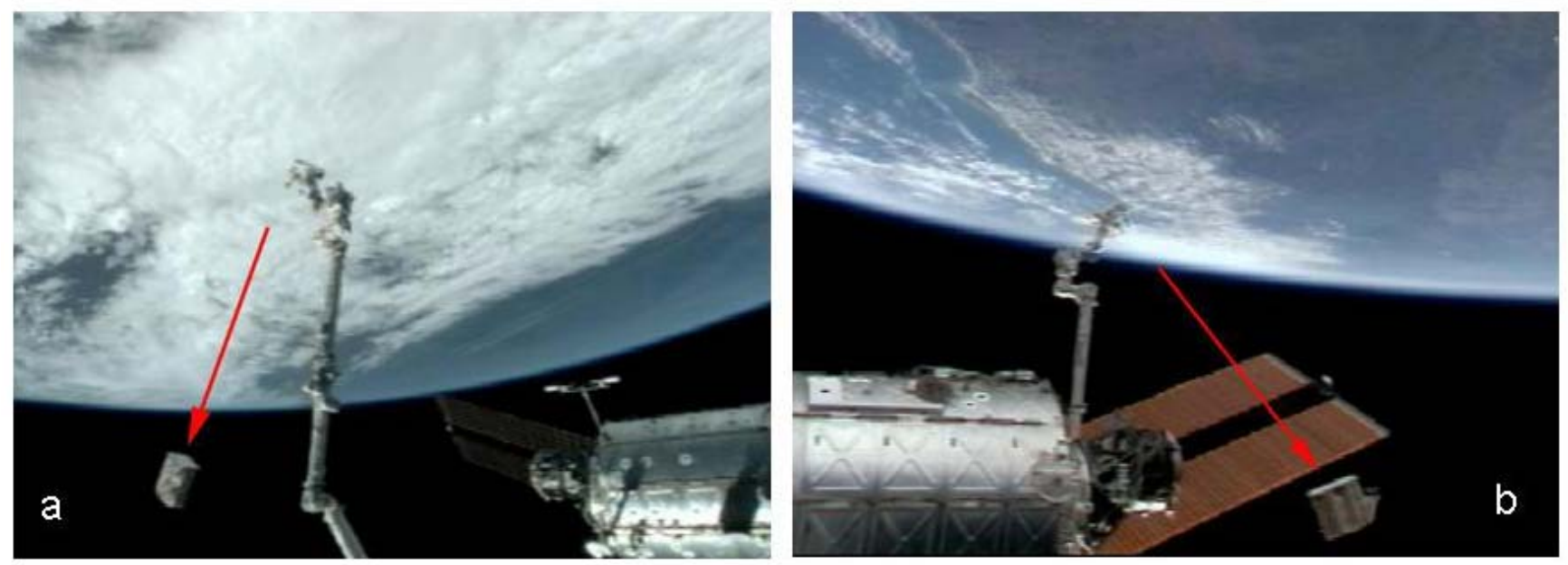

Fig. 11. EAS jettison starboard (a) and port (b) camera views.

Red arrows indicate EAS motion in the camera field of view.

Quick-look velocity estimations were done with the intersection method (section 3) and provided within one hour after the video-clips were downlinked and recorded in the Image Science and Analysis Laboratory (ISAL) at JSC. It was immediately obvious that the $\mathrm{X}$-component of the velocity is large enough to provide the safe departure, however, a large Z-component (see Table 2) and a large margin of error of the Quick-look estimation raised some concerns. The results delivered in several hours (during and immediately after the EVA) proved that the velocity vector was in the safety margins (actually, along the lateral surface of the safety acceptance cone) and that even one planned reboost would insure the ISS safety.

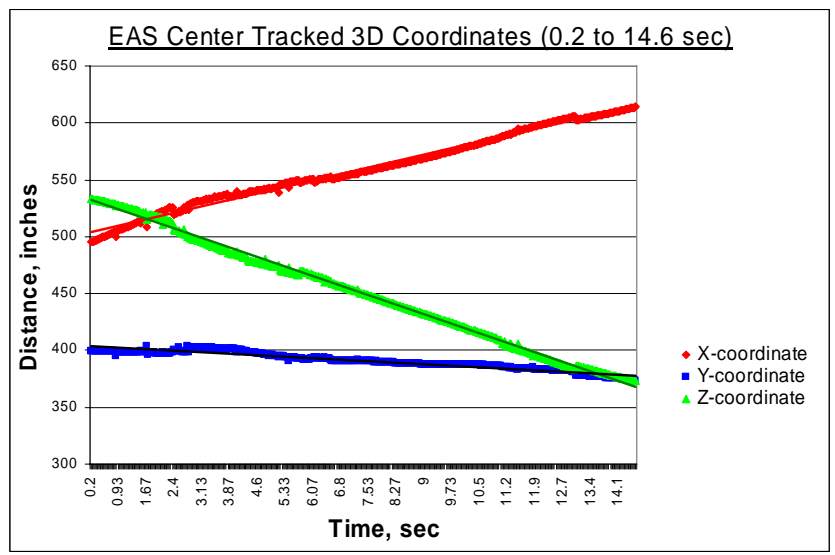


Tracking Number: DSS08-DS33-1, Paper Number: 6958-17

Fig. 12. Example of the EAS center position computation from on a part of its trajectory. 
Table 2. EAS velocity vector measurements results in the ISSACS.

\begin{tabular}{|c|c|c|c|}
\hline Analyses: & $\begin{array}{c}\text { Quick Look } \\
\text { (delivered in } \\
1-2 \text { hours) }\end{array}$ & $\begin{array}{c}\text { Real Time } \\
\text { (delivered in } \\
4-5 \text { hours) }\end{array}$ & $\begin{array}{c}\text { Final Report } \\
\text { (next day) }\end{array}$ \\
\hline $\mathrm{V}_{\mathrm{X}} \mathrm{cm} / \mathrm{sec}$ & $19 \pm 8$ & $21.4 \pm 4$ & $20.5 \pm 3$ \\
\hline $\mathrm{V}_{\mathrm{Y}} \mathrm{cm} / \mathrm{sec}$ & $-3 \pm 8$ & $-5.8 \pm 4$ & $-5.3 \pm 3$ \\
\hline $\mathrm{V}_{\mathrm{Z}} \mathrm{cm} / \mathrm{sec}$ & $-26 \pm 8$ & $-26.1 \pm 4$ & $-27.6 \pm 3$ \\
\hline
\end{tabular}

\subsection{Video Stanchion Support Assembly}

The Video Stanchion Support Assembly (VSSA) [Support Equipment] had to be discarded to clear the External Storage Platform (ESP) so that it could receive new equipment. Its mass was $96 \mathrm{~kg}$ and its dimensions, $1.2 \mathrm{x} 1.3 \mathrm{x} 0.9 \mathrm{~m}$. VSSA was jettisoned during the same EVA as the EAS and before it. The reason was that the VSSA was easier to remove and jettison because it was smaller and lighter than the EAS. It also provided some practice for Clay Anderson before the EAS jettison (which was much harder to push, as he characterized it afterwards). Results of the measurements made in the ISAL (Table 3) show that the VSSA departure velocity was approximately five times larger than that of the EAS, however, it has also a large component in the Zenith direction (Z-coordinate of the ISACS).

Shown below are images taken from the port and starboard truss cameras and the velocity measurements results. Note, that the VSSA jettison took place shortly after "sunrise" and it was more difficult to acquire imagery due to fast changing illumination. The EAS jettison occurred well after the "sunrise" that guaranteed high quality imagery for the photogrammetry analysis (compare Figs. 11 and 13).
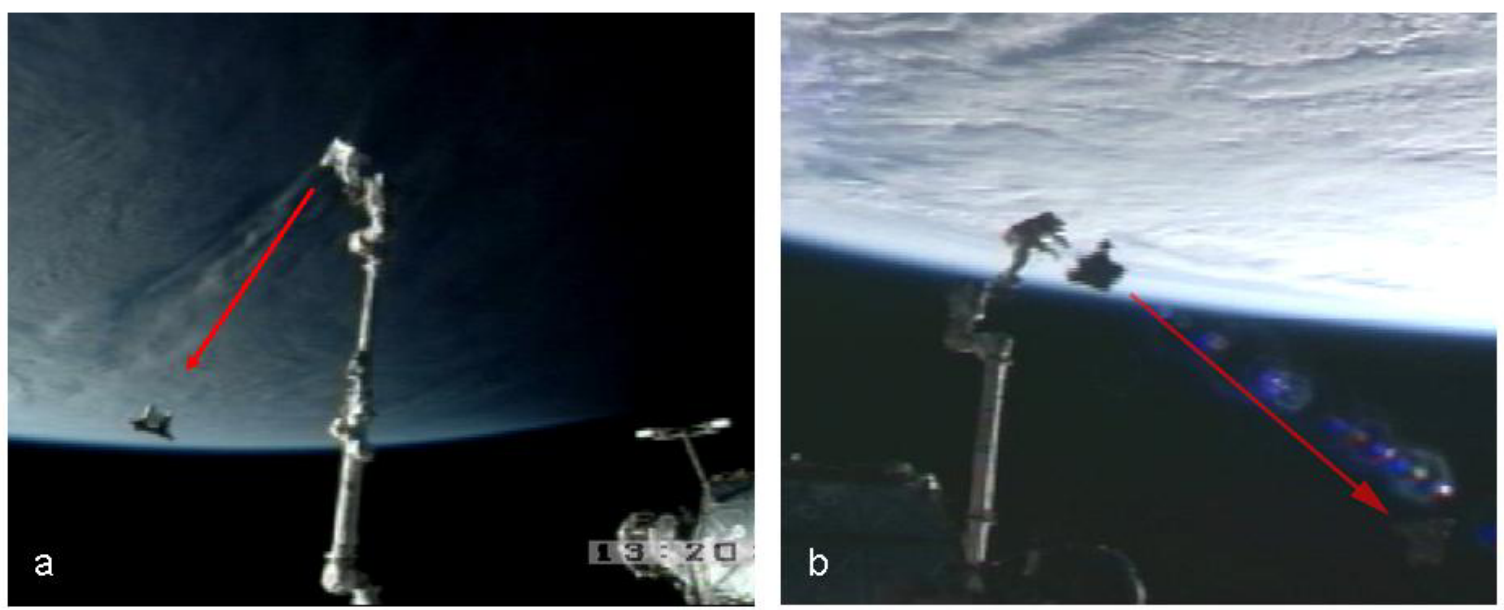

Fig. 13. VSSA jettison starboard (a) and port (b) camera views. Red arrows indicate VSSA motion in the camera field of view.

Table 3. VSSA velocity vector measurements results in the ISSACS system of coordinates.

\begin{tabular}{|c|c|c|c|}
\hline Analyses: & $\begin{array}{c}\text { Quick Look } \\
\text { (delivered in } \\
3-4 \text { hours)* }\end{array}$ & $\begin{array}{c}\text { Real Time } \\
\text { (delivered in } \\
4-5 \text { hours) }\end{array}$ & $\begin{array}{c}\text { Final Report } \\
\text { (next day) }\end{array}$ \\
\hline $\mathrm{V}_{\mathrm{X}} \mathrm{cm} / \mathrm{sec}$ & $90 \pm 10$ & $101 \pm 8$ & $102 \pm 5$ \\
\hline $\mathrm{V}_{\mathrm{Y}} \mathrm{cm} / \mathrm{sec}$ & $-6 \pm 10$ & $-7 \pm 8$ & $-11 \pm 5$ \\
\hline $\mathrm{V}_{\mathrm{Z}} \mathrm{cm} / \mathrm{sec}$ & $-70 \pm 10$ & $-81 \pm 8$ & $-86 \pm 5$ \\
\hline
\end{tabular}

*) Camera orientations had to be derived from the next jettison (EAS) data. 


\section{CONCLUSION}

We presented here a photogrammetry approach to the task of evaluating trajectory and velocity vector of the objects jettisoned from the ISS in real time. It is shown that this method is a reliable and unique tool for operational evaluation of a possible safety hazard. Timely estimation of the jettisoned object trajectory makes it possible to maintain adequate situation awareness pending ground-based tracking.

Difficulties of implementation of this method originate due to lack of multiple reference targets in space during on-orbit operations. Accuracy of the trajectory computations is much less than when implementing on-the-ground close-range photogrammetry. Nevertheless, the velocity vector computations are more accurate than any other means available, and they produce extremely important information relating to ISS safety.

\section{ACKNOWLEDGMENTS}

This work has been accomplished in support of the International Space Station Program at NASA Johnson Space Center. The authors would like to thank Michael Snyder, Robert Scharf, Donn Liddle, Eric Nielsen and other members of the Image Science and Analysis Group for help. We also thank the Trajectory Operations Team (TOPO) for support of the Photogrammetry tasks.

\section{REFERENCES}

1. http://www.nasa.gov/astronauts/anderson_profile.html

2. Close Range Photogrammetry and Machine Vision, K.B. Atkinson editor, Wittles Publ., 1996, p. 25.

3. E.M. Mikhail, J.S. Bethel, and J.C. McGlone, Introduction to Modern Photogrammetry, J. Wiley, NY-London, 2001

4. http://www.nasa.gov/mission_pages/station/expeditions/expedition12/exp12_eva.html

5. http://www.trackeye.com/ImageSystems/TrackEyeproduct.html

6. http://www.nasa.gov/mission_pages/station/expeditions/expedition12/exp12_eva2.html

7. http://www.nasa.gov/mission_pages/station/expeditions/expedition15/e15_eva_072307_prt.htm

8. http://dx12.jsc.nasa.gov/about/SCTF.shtml 